\title{
High-precision acoustic helium signatures in 18 low-mass low-Iuminosity red giants
}

\section{Analysis from more than four years of Kepler observations ${ }^{\star}$}

\author{
E. Corsaro ${ }^{1,2,3,4}$, J. De Ridder ${ }^{4}$, and R. A. García ${ }^{3}$ \\ 1 Instituto de Astrofísica de Canarias, 38205 La Laguna, Tenerife, Spain \\ 2 Universidad de La Laguna, Departamento de Astrofísica, 38206 La Laguna, Tenerife, Spain \\ ${ }^{3}$ Laboratoire AIM, CEA/DSM - CNRS - Univ. Paris Diderot - IRFU/SAp, Centre de Saclay, 91191 Gif-sur-Yvette Cedex, France \\ e-mail: enrico.corsaro@cea.fr \\ ${ }^{4}$ Instituut voor Sterrenkunde, KU Leuven, Celestijnenlaan 200D, 3001 Leuven, Belgium
}

Received 18 February 2015 / Accepted 13 April 2015

\section{ABSTRACT}

\begin{abstract}
Context. High-precision frequencies of acoustic modes in red giant stars are now available thanks to the long observing length and high quality of the light curves provided by the NASA Kepler mission, thus allowing the interior of evolved cool low-mass stars to be probed with an unprecedented level of detail.

Aims. We characterize the acoustic signature of the helium second ionization zone in a sample of 18 low-mass low-luminosity red giants by exploiting new mode-frequency measurements derived from more than four years of Kepler observations.

Methods. We analyzed the second frequency differences of radial acoustic modes in all the stars of the sample by using the Bayesian code DIAMONDS.

Results. We find clear acoustic glitches due to the signature of helium second ionization in all the stars of the sample. We could measure the acoustic depth and the characteristic width of the acoustic glitches with a precision level on average around $\sim 2 \%$ and $\sim 8 \%$, respectively. We find good agreement with theoretical predictions and existing measurements from the literature. Finally, we derive the amplitude of the glitch signal at $v_{\max }$ for the second differences and for the frequencies with an average precision of $\sim 6 \%$, obtaining values in the range $0.14-0.24 \mu \mathrm{Hz}$ and $0.08-0.33 \mu \mathrm{Hz}$, respectively, which can be used to investigate the helium abundance in the stars.
\end{abstract}

Key words. asteroseismology - stars: oscillations - stars: late-type - stars: interiors - methods: statistical - methods: data analysis

\section{Introduction}

The so-called acoustic glitches are regions of sharp-structure variation located in the interior of stars and caused by the presence of a change in the energy transport from radiative to convective, by a rapid variation in the chemical composition, or by ionization zones of chemical elements, such as hydrogen and helium. As originally predicted for the Sun (e.g., Vorontsov 1988; Gough 1990), these regions produce tiny and regular variations in the frequency of the acoustic $(p)$ modes that can be detected by direct measurement of the characteristic large frequency separation, namely the frequency separation between modes having the same angular degree.

By studying the glitch signature in the Sun, it was possible to measure the acoustic position of the base of the convective zone and of the helium second ionization (He II) zone. The signature can also be used to provide estimates of the helium abundance in the envelope and the extent of the overshooting (e.g., Basu \& Antia 1995; Basu 1997; Monteiro \& Thompson 2005; Christensen-Dalsgaard et al. 2011). The same effect was expected to be observed in distant stars (e.g., Monteiro et al. 2000; Mazumdar \& Antia 2001; Ballot et al. 2004). Thanks to the advent of the CoRoT (Baglin et al. 2006) and Kepler space

\footnotetext{
* Appendix $\mathrm{A}$ is available in electronic form at http: //www . aanda . org
}

missions (Borucki et al. 2010; Koch et al. 2010), an outstanding number of high-quality photometric observations for thousands of stars has been released. This yielded the frequency shifts caused by the acoustic glitches to be discovered and analyzed in many low-mass, main-sequence, subgiant and red-giant stars (RGs) (Mosser et al. 2010; Miglio et al. 2010; Mazumdar et al. 2012, 2014; Verma et al. 2014), allowing the positions of the base of the convective zone, of the He II zone in main-sequence and subgiant stars, and of the He II zone in the case of the red giants to be constrained.

The asteroseismology of red giant stars, in particular, has led to several important breakthroughs in the stellar physics of lowmass stars in recent years (e.g. Beck et al. 2011; Mosser et al. 2011a; Bedding et al. 2011; Beck et al. 2012; Deheuvels et al. 2012). The characterization of the glitch signatures is able to provide tighter constraints on the chemical composition and the internal stratification of the star, and potentially allows retrieving helium abundances in distant stars, essential for population studies (e.g., see Broomhall et al. 2014, hereafter B14, and references therein). More recent studies focusing on these evolved cool stars have analyzed the glitches due to the He II zone for more than a hundred targets observed by Kepler (Vrard et al. 2014), and thoroughly investigated the properties of the signature from a theoretical point of view (B14, see also Christensen-Dalsgaard et al. 2014, for more discussion). 
The recent availability of Kepler datasets spanning more than four years of nearly continuous observations, coupled with the development of new computational advances in asteroseismic data analysis (e.g., see Corsaro \& De Ridder 2014; Corsaro et al. 2015, hereafter C15), enables the study of the acoustic glitch signatures in red giants with an unprecedented level of detail.

In this paper, we report on the evidence of clear acoustic glitch signatures due to the He II zone in the sample of 19 red giants recently investigated by $\mathrm{C} 15$, so we fully characterize the oscillatory signal by means of a Bayesian approach.

\section{Data analysis}

As noticed by $\mathrm{C} 15$, the low-mass low-luminosity red giants (LRGs) are candidates that are well suited to testing stellar structure models and stellar evolution theory. The less-evolved stage in the red giant branch (RGB) of the stellar evolution for the LRGs implies the highest frequency of maximum power $v_{\max }$ for a red giant (between 100 and $200 \mu \mathrm{Hz}$ ), hence a broader power excess caused by the oscillations and, consequently, a larger number of radial orders observed (in general between six and nine). By having a larger number of high signal-to-noise ratio $p$-mode frequencies available, one is thus able to constrain the signature of the glitches more efficiently.

In this work we analyze the sample of LRGs studied by $\mathrm{C} 15$, who fitted and characterized their full oscillation spectrum using Kepler observations from Q0 till Q17.1, a total of $\sim 1470$ days, with a frequency resolution of $\delta v_{\text {bin }} \simeq 0.008 \mu \mathrm{Hz}$. The stars have $v_{\max }$ values ranging from 110 to $\sim 190 \mu \mathrm{Hz}$ and masses in the interval $1-2 M_{\odot}$. We adopt the high-precision individual frequency measurements from $\mathrm{C} 15$, and follow the theoretical approach of B14.

In the present analysis we refer to the first (frequency) difference as the large frequency separation of a given angular degree, which is a function of the frequency in the power spectral density of the star, $\Delta v_{\ell}(v)$. For a radial order $n, \Delta v_{\ell}(v)$ is thus defined as

$\Delta v_{n, \ell} \equiv v_{n+1, \ell}-v_{n, \ell}$,

where $v_{n, \ell}$ is the central frequency of the mode with angular degree $\ell$ and radial order $n$. In addition, we compute the second (frequency) difference (see, e.g., Gough 1990), $\Delta_{2} v_{\ell}(v)$, defined for a single radial order as

$$
\begin{aligned}
\Delta_{2} v_{n, \ell} & \equiv v_{n+1, \ell}-2 v_{n, \ell}+v_{n-1, \ell} \\
& =\Delta v_{n, \ell}-\Delta v_{n-1, \ell} .
\end{aligned}
$$

We fit the acoustic glitch signatures with the model introduced by Houdek \& Gough (2007) and used by B14 for RGs, defined as

$$
\Delta_{2} \omega_{n, \ell}=A \omega_{n, \ell} \exp \left(-2 b^{2} \omega_{n, \ell}^{2}\right) \cos \left[2\left(\tau_{\mathrm{He} \mathrm{II}} \omega_{n, \ell}+\phi\right)\right]+c,
$$

with $\omega_{n, \ell} \equiv 2 \pi v_{n, \ell}$ and $\Delta_{2} \omega_{n, \ell} \equiv 2 \pi \Delta_{2} v_{n, \ell}, A$ a dimensionless amplitude of the signature signal, $\tau_{\mathrm{He}}$ the acoustic depth of the He II zone, $b$ its characteristic width, and $\phi$ and $c$ are constant phase shift and offset, respectively, of the oscillatory signal. Following the arguments discussed by B14, we apply the fit to the second differences only because they are less prone to additional varying components, such as hydrogen ionization and non-adiabatic processes, and to the general frequency dependence of the large separation caused by the development of the second-order term of the asymptotic relation (Mosser et al. 2011b). The second differences are at the same time available in a reasonably high number of measurements (two less than the total number of modes obtained for a given angular degree), still allowing the model parameters to be constrained without leading to degeneracies in the solutions.

Despite the possible presence of the oscillatory component in modes of angular degree $\ell>0$, we point out that only radial mode frequencies are used for the final fit. The reason behind this choice is mainly the need to exploit pure $p$-mode character oscillations (see also B14), which in the case of RGs, are only available in the form of $\ell=0$ modes. This is because the coupling occurring between $p$ modes of angular degree $\ell>0$ and $g$ modes arising from the radiative interior can hamper the asymptotic behavior of the corresponding modes by producing so-called mixed modes, whose frequencies deviate from the expected position of a pure $p$ mode oscillation (e.g., Beck et al. 2011).

We perform all the fits following a Bayesian approach using DiAMONDS (Corsaro \& De Ridder 2014), hence exploiting a nested-sampling Monte Carlo method to perform the inference and estimate the free parameters of the model given by Eq. (3) from their individual marginal probability distributions (see Corsaro \& De Ridder 2014, for more details). The configuring parameters of DIAMONDS (following the definitions by Corsaro \& De Ridder 2014) used for all the computations are initial enlargement fraction $1.0 \leq f_{0} \leq 1.7$, shrinking rate $\alpha=0.02-0.03$, number of live points $N_{\text {live }}=1000$, number of clusters $1 \leq N_{\text {clust }} \leq 4$, number of total drawing attempts $M_{\text {attempts }}=10^{4}$, number of nested iterations before the first clustering $M_{\text {init }}=1000$, and number of nested iterations with the same clustering $M_{\text {same }}=50$.

For this analysis we adopted a normal likelihood function, such as the one used by Corsaro et al. (2013), which takes the uncertainties into account, with correlations included, on the measurements of the second differences. This assumes that the residuals arising from the difference between predicted and measured second differences are Gaussian-distributed. Since we did not have any initial guesses available from the literature for the given stars for the set up of priors, we used uniform (i.e., flat) prior probability distributions for all the free parameters of Eq. (3), with lower and upper boundaries for each parameter range obtained by comparison with existing measurements of the acoustic depths derived by Miglio et al. (2010), Mazumdar et al. (2014), and the theoretical results by B14 in the observed range of $v_{\max }$. The choice of uniform priors also yielded a faster computation with DiAMONDS, as already discussed by Corsaro \& De Ridder (2014) and C15.

Following the discussion by Ballot et al. (2004) and B14, we computed the acoustic radius of the He II zones, $t_{\mathrm{He}}$ II , since it represents a quasi unbiased estimator of the acoustic position of the glitch. This is done by using the mean large frequency separation, $\langle\Delta v\rangle$, obtained from the radial mode frequencies provided by $\mathrm{C} 15$, giving the total acoustic radius of the star, $T=(2\langle\Delta v\rangle)^{-1}$, hence the acoustic radius of the He II zone, $t_{\mathrm{He} \text { II }} \equiv T-\tau_{\mathrm{He} \mathrm{II}}$.

Finally, to provide measurements that can be used to model the helium abundance in the envelope of the stars, following B14, we extracted the amplitude of the signal at $v_{\max }$ from Eq. (3), obtaining

$A_{\max }=A v_{\max } \exp \left(-2 b^{2} \omega_{\max }^{2}\right)$,

with $\omega_{\max }=2 \pi v_{\max }$, and $v_{\max }$ derived from the background fit done by C15. Following Verma et al. (2014), we also derived the 
amplitude of the signal in the frequencies, $A_{\mathrm{He}}$, given as

$A_{\mathrm{He}}=\frac{A_{\max }}{4 \cos ^{2}\left[2 \pi \tau_{\mathrm{He}}\langle\Delta v\rangle\right]}$,

where $\langle\Delta v\rangle$ is the same mean large frequency separation used to calculate $t_{\mathrm{He} \mathrm{II}}$, and $\tau_{\mathrm{He} \mathrm{II}}$ is the same acoustic depth used in Eq. (3). The adoption of $A_{\mathrm{He}}$ to retrieve the helium content in the envelope was preferred since this value is not influenced by the location of the glitch (see, e.g., Mazumdar \& Antia 2001; Mazumdar et al. 2014; Verma et al. 2014). For clarity, we stress that $A_{\max }$ is derived from a Bayesian approach by using the same sampling of the posterior probability distribution obtained by DIAMONDS for the free parameters of the glitch model (see also Corsaro \& De Ridder 2014, Fig. 7, for an analogous case presenting the sampling from DIAMONDS for a combination of different inferred parameters). The parameters $t_{\mathrm{He}}$ II and $A_{\mathrm{He}}$ simply follow from their definitions as presented above, by using both the value $\langle\Delta v\rangle$, computed from the radial mode frequencies presented by $\mathrm{C} 15$ for each star of our sample, and the estimated model parameters of Eq. (3) (see Sect. 3 for more details).

\section{Results}

The results for $\Delta v_{\ell}(v)$ and $\Delta_{2} v_{\ell}(v)$ for the star KIC 12008916 are shown in Fig. 1 (top and bottom panels, respectively) and can be found in Appendix A for all the other LRGs, together with the tables with the individual measurements of the radial angular frequencies $\omega_{n, 0}$, and of the corresponding second angular frequency differences $\Delta_{2} \omega_{n, 0}(v)$ used in this work. We discarded KIC 10123207 from the fit because of the low number of available measurements (foursecond differences only, one less than the minimum required to fit the model given by Eq. (3)). To help the reader visualize the presence of the oscillatory signal in the first differences of the angular degrees $\ell=0,2,3$ and in the second differences of the angular degrees $\ell=2,3$, we included low-degree (3-4) polynomial fits. The $1 \sigma$ uncertainties on the first and second differences derived from a standard error propagation of the uncertainties of the individual mode frequencies, following Eqs. (1) and (2), respectively, are overlaid in each plot, though they are not visible in most of the cases because they are smaller than the size of the symbols used for the measurements. The uncertainties on all the measurements are listed in the corresponding tables in Appendix A for completeness.

We find that all the stars we analyzed have clear acoustic glitches due to the signature of the He II zone in $\ell=0$ and 2 modes up to the second differences. We can also see the presence of acoustic glitches in $\ell=3$ modes for most of the stars thanks to at least four different frequency measurements that are available. As mentioned in Sect. 2, we find that the measurements for modes having angular degrees $\ell=2,3$ often deviate from those of the radial oscillations (see, e.g. Figs., A.6, A.8, A.10, and A.13). As also indicated by $\mathrm{C} 15$ for the case of the mode linewidths of the $\ell=2$ modes, this different behavior relies on the presence of both mixed quadrupole modes and rotational split components. When using an individual Lorentzian profile to fit the frequency region containing the oscillation peak, either an $\ell=2$ or 3 mode, as done by $\mathrm{C} 15$, the effects mentioned before can significantly change the measured frequency of the peak. A reliable treatment of the mixed modes and of the rotational split components for $\ell=2,3$ modes is, however, difficult due to the high proximity of the individual peaks.

The model fit to the acoustic glitch signatures of KIC 12008916 is shown in the bottom panel of Fig. 1 for

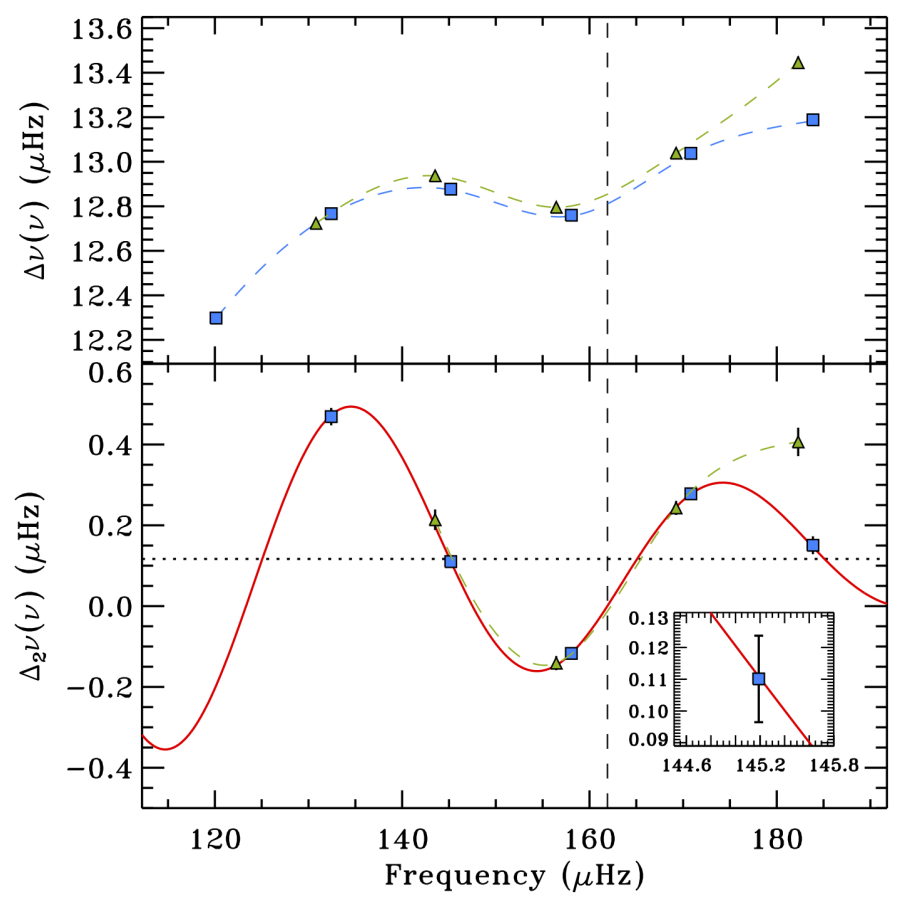

Fig. 1. Acoustic glitches for KIC 12008916. Top panel: the first difference, $\Delta v(v)$, Eq. (1). Blue squares represent values computed from $\ell=0$ modes, while green triangles from $\ell=2$ modes, with polynomial fits (dashed lines with same color as symbols) overlaid to visualize the oscillatory trend. The dashed vertical line marks the position of $v_{\max }$ for a reference. Bottom panel: the second difference $\Delta_{2} v(v)$, Eq. (2), with the same symbol description as for the top panel. The solid red line indicates the fit to the $\ell=0$ measurements given by Eq. (3) with the estimated parameters listed in Table 1, as derived by DIAMONDS. The horizontal dotted line denotes the offset level $c /(2 \pi)$, which is useful for visualizing the amplitude of the signature. The inset shows a zoom in of one of the measurements to visualize the precision level of the fit.

the case of $\Delta_{2} v_{n, 0}(v)$, and similarly for the other stars in Appendix A. All the estimated parameters of Eq. (3) are provided in Table 1. The inset in the bottom panel of Fig. 1 provides a closer view of one of the measurements for visualizing the precision-level achieved in the fit. In particular, we find that the model given by Eq. (3) yields a remarkable fit quality for most of the stars, with average uncertainties of $\sim 2 \%$ for $\tau_{\mathrm{He} \text { II }}$ and $\sim 8 \%$ for $b$. Following the analysis presented by Corsaro et al. (2013), we obtained the weighted Gaussian rms of the residuals, $\sigma_{\text {rms }}$ (listed in Table 1 as well).

To computate the rms, we adopted the weights $w_{i}=\sigma_{i}^{-2}, \sigma_{i}$, which are the uncertainties on the second frequency difference coming from those reported in Tables A.1. The quantity $\sigma_{\text {rms }}$ provides additional information to the reader because it allows the quality of the fits to be compared between different stars and the precision achieved on the individual fits to the given uncertainties of the data points to be related. We note that for all the fits presented in this work, the values for $\sigma_{\text {rms }}$ are remarkably low, ranging from $10^{-2}$ down to $10^{-3} \mu \mathrm{Hz}$ in the best cases, thus in many cases reaching the same order of the precision level obtained on the individual frequencies of the radial modes. For a reference to the reader, in Table 1 we also provide the values for the total acoustic radius $T$, with its $1 \sigma$ standard deviation and the values of $v_{\max }$ obtained by $\mathrm{C} 15$.

The stars KIC 8475025, KIC 9145955, KIC 10200377, and KIC 11913545 each show a component at high frequency that is not properly predicted by the adopted model. This mainly 
Table 1. Median values with corresponding 68.3\% shortest credible intervals as derived by DIAMONDS for the parameters discussed in Sect. 2.

\begin{tabular}{|c|c|c|c|c|c|c|c|c|c|c|c|}
\hline KIC ID & $\begin{array}{c}\tau_{\mathrm{He} \mathrm{II}} \\
(\mathrm{s})\end{array}$ & $A$ & $\begin{array}{c}b \\
(\mathrm{~s})\end{array}$ & $\begin{array}{c}\phi \\
(\mathrm{rad})\end{array}$ & $\begin{array}{c}c \\
(\mu \mathrm{Hz})\end{array}$ & $\begin{array}{c}t_{\mathrm{He} \mathrm{II}} \\
(\mathrm{s})\end{array}$ & $\begin{array}{c}T \\
\text { (s) }\end{array}$ & $\begin{array}{c}A_{\max } \\
(\mu \mathrm{Hz})\end{array}$ & $\begin{array}{c}A_{\mathrm{He}} \\
(\mu \mathrm{Hz})\end{array}$ & $\begin{array}{c}v_{\max } \\
(\mu \mathrm{Hz})\end{array}$ & $\begin{array}{c}\sigma_{\mathrm{rms}} \\
(\mu \mathrm{Hz})\end{array}$ \\
\hline 03744043 & $13468_{-272}^{+258}$ & $0.0131_{-0.0015}^{+0.0010}$ & $1535_{-42}^{+27}$ & $1.9_{-0.2}^{+0.2}$ & $0.86_{-0.03}^{+0.03}$ & $37183_{-638}^{+643}$ & $50652_{-584}^{+584}$ & $0.139_{-0.005}^{+0.004}$ & $0.077_{-0.003}^{+0.002}$ & 112.52 & 0.007 \\
\hline 06117517 & $18422_{-263}^{+303}$ & $.0403_{-0.0}^{+0.0}$ & $1685_{-155}^{+153}$ & $0.7_{-0.2}^{+0.2}$ & $0.58_{-0.05}^{+0.04}$ & $31128_{-563}^{+543}$ & $49551_{-475}^{+475}$ & $0.188_{-0.028}^{+0.021}$ & $0.306_{-0.046}^{+0.034}$ & 120.27 & 0.017 \\
\hline 06144777 & $16083_{-230}^{+228}$ & $0.0034_{-0.0007}^{+0.0006}$ & $720_{-107}^{+106}$ & $1.4_{-0.2}^{+0.2}$ & $0.67_{-0.03}^{+0.02}$ & $29576_{-448}^{+449}$ & $45660_{-386}^{+386}$ & $0.220_{-0.007}^{+0.007}$ & $0.274_{-0.009}^{+0.009}$ & 129.69 & 0.011 \\
\hline 07060732 & $15886_{-205}^{+205}$ & $0.0142_{-0.0027}^{+0.0023}$ & $1256_{-65}^{+64}$ & $1.4_{-0.2}^{+0.2}$ & $0.65_{-0.03}^{+0.04}$ & $30032_{-482}^{+480}$ & $45919_{-436}^{+436}$ & $0.212_{-0.011}^{+0.013}$ & $0.245_{-0.013}^{+0.015}$ & 132.29 & 0.017 \\
\hline 07619745 & $12231_{-227}^{+240}$ & $0.0131_{-0.0048}^{+0.0004}$ & $1041_{-82}^{+80}$ & $1.5_{-0.2}^{+0.2}$ & $0.63_{-0.04}^{+0.04}$ & $26064_{-391}^{+383}$ & $38296_{-309}^{+309}$ & $0.184_{-0.013}^{+0.011}$ & $0.159_{-0.011}^{+0.013}$ & 170.82 & 0.011 \\
\hline 08366239 & $11826_{-288}^{+271}$ & $0.0118_{-0.0029}^{+0.0022}$ & $940_{-51}^{+53}$ & $1.0_{-0.3}^{+0.2}$ & $0.49_{-0.05}^{+0.06}$ & $24686_{-397}^{+409}$ & $36513_{-291}^{+291}$ & $0.196_{-0.014}^{+0.013}$ & $0.177_{-0.013}^{+0.011}$ & 185.56 & 0.020 \\
\hline 08475025 & $17796_{-283}^{+280}$ & $0.0065_{-0.0024}^{+0.0017}$ & $1121_{-122}^{+169}$ & $2.0_{-0.2}^{+0.3}$ & $0.61_{-0.05}^{+0.05}$ & $34406_{-533}^{+535}$ & $52203_{-454}^{+454}$ & $0.207_{-0.013}^{+0.014}$ & $0.225_{-0.014}^{+0.013}$ & 112.95 & 0.023 \\
\hline 08718745 & $12468_{-415}^{+434}$ & $0.0120_{-0.0021}^{+0.0018}$ & $1266_{-53}^{+53}$ & $1.3_{-0.3}^{+0.3}$ & $1.02_{-0.05}^{+0.04}$ & $31490_{-686}^{+674}$ & $43958_{-531}^{+531}$ & $0.187_{-0.007}^{+0.009}$ & $0.118_{-0.004}^{+0.006}$ & 129.31 & 0.012 \\
\hline 09145955 & $15626_{-274}^{+271}$ & $0.0100_{-0.0034}^{+0.00025}$ & $1232_{-107}^{+109}$ & $1.7_{-0.2}^{+0.3}$ & $0.60_{-0.04}^{+0.04}$ & $29712_{-502}^{+580}$ & $45339_{-424}^{+424}$ & $0.164_{-0.013}^{+0.0012}$ & $0.186_{-0.015}^{+0.004}$ & 131.65 & 0.015 \\
\hline 0926 & $15536_{-430}^{+410}$ & $0.0072_{-0.0018}^{+0.0014}$ & $1155_{-93}^{+100}$ & $3.0_{-0.3}^{+0.2}$ & $0.87_{-0.04}^{+0.04}$ & $32891_{-680}^{+693}$ & $48427_{-544}^{+544}$ & $0.192_{-0.007}^{+0.008}$ & $0.169_{-0.006}^{+0.007}$ & 118.63 & 0.018 \\
\hline 09475697 & $16782_{-296}^{+304}$ & $0.0060_{-0.0016}^{+0.0012}$ & $1070_{-111}^{+117}$ & $2.5_{-0.2}^{+0.2}$ & $0.65_{-0.04}^{+0.03}$ & $34052_{-595}^{+590}$ & $50835_{-512}^{+512}$ & $0.207_{-0.008}^{+0.008}$ & $0.200_{-0.008}^{+0.008}$ & 115.05 & 0.015 \\
\hline 09882316 & $9232_{-475}^{+356}$ & $0.0342_{-0.0083}^{+0.00071}$ & $1176_{-46}^{+39}$ & $4.1_{-0.4}^{+0.2}$ & $0.73_{-0.06}^{-0.04}$ & $27402_{-429}^{+532}$ & $36634_{-240}^{+240}$ & $0.163_{-0.014}^{+0.008}$ & $0.083_{-0.007}^{+0.007}$ & 182.04 & 0.011 \\
\hline 10200377 & $15660_{-645}^{+626}$ & $0.0415_{-0.0088}^{+0.0063}$ & $1517_{-70}^{+60}$ & $0.9_{-0.6}^{+0.4}$ & $0.76_{-0.03}^{+0.04}$ & $24327_{-704}^{+721}$ & $39988_{-323}^{+323}$ & $0.147_{-0.024}^{+0.014}$ & $0.330_{-0.054}^{+0.038}$ & 2.52 & 0.029 \\
\hline 10257278 & $10565_{-353}^{+440}$ & $0.1106_{-0.0155}^{+0.0180}$ & $1639_{-29}^{+32}$ & $1.5_{-0.4}^{+0.0}$ & $0.83_{-0.05}^{+0.05}$ & $30551_{-574}^{+510}$ & $41116_{-369}^{+369}$ & $0.144_{-0.008}^{+0.007}$ & $0.075_{-0.004}^{+0.004}$ & 149.47 & 0.007 \\
\hline 11353313 & $16819_{-447}^{+388}$ & $0.0104_{-0.0053}^{+0.0038}$ & $1255_{-171}^{+160}$ & $1.4_{-0.3}^{+0.4}$ & $0.71_{-0.05}^{+0.04}$ & $29749_{-597}^{+637}$ & $46568_{-455}^{+455}$ & $0.178_{-0.011}^{+0.014}$ & $0.249_{-0.015}^{+0.020}$ & 126.46 & 0.006 \\
\hline 11913545 & $15218_{-482}^{+338}$ & $0.0122_{-0.0034}^{+0.00035}$ & $1412_{-95}^{+81}$ & $3.4_{-0.3}^{+0.3}$ & $0.70_{-0.03}^{+0.03}$ & $34222_{-601}^{+691}$ & $49440_{-498}^{+498}$ & $0.164_{-0.006}^{+0.006}$ & $0.127_{-0.005}^{+0.005}$ & 117.16 & 0.005 \\
\hline 11968334 & $13429_{-313}^{+294}$ & $0.0074_{-0.0011}^{+0.0012}$ & $987_{-61}^{+68}$ & $2.6_{-0.3}^{+0.3}$ & $0.72_{-0.04}^{+0.04}$ & $30573_{-529}^{+540}$ & $44003_{-441}^{+441}$ & $0.223_{-0.009}^{+0.007}$ & $0.169_{-0.007}^{+0.005}$ & 141.43 & 0.007 \\
\hline 12008916 & $12492_{-302}^{+286}$ & $0.0113_{-0.0028}^{+0.0023}$ & $987_{-56}^{+63}$ & $2.0_{-0.3}^{+0.3}$ & $0.73_{-0.05}^{+0.04}$ & $26505_{-474}^{+484}$ & $38998_{-379}^{+379}$ & $0.243_{-0.011}^{+0.007}$ & $0.212_{-0.010}^{+0.006}$ & 161.92 & 0.013 \\
\hline
\end{tabular}

Notes. The parameters refer to the angular measurements of the second differences, $\Delta_{2} \omega_{n, 0}(v)$, and the corresponding frequencies $\omega_{n, 0}$, of only the radial modes. The parameters of the model to fit the acoustic glitch signatures in the second differences, Eq. (3), correspond to the columns from one to six. The acoustic radius of the He II zone, the total acoustic radius of the star, and the amplitude at $v_{\max }$ in the second difference and in frequency (Eqs. (4) and (5), respectively) are indicated in the columns from seven to ten. The last two columns provide the reference values for $v_{\max }$ (provided by $\mathrm{C} 15$ ) and the weighted Gaussian rms of the residuals, as described in Sect. 3.

relies on some residual frequency dependence of the second differences that becomes more pronounced towards the wings of the region containing the oscillations. However, we note that the measurements at higher frequencies all have larger error bars (up to 10 times) with respect to the others, because of the larger linewidths of the peaks occurring at high frequency (see C15 for more details). The fits derived, except for KIC 8475025, are therefore not significantly affected by the measurements of the second differences falling at high-frequency, whereas they are almost entirely constrained by those close to $v_{\max }$. This is inspected by refitting the glitch model without the highest frequency measurement of the second difference (showing the deviating behavior), hence noticing that the new estimated free parameters of the model lie well within the uncertainties of those reported in Table 1. For KIC 8475025, however, we find that the fit is unstable owing to the large deviation (more than $0.2 \mu \mathrm{Hz}$ ) of the second difference measurement falling at the highest frequency (see Fig. A.7). This is because the measured oscillation frequency of the highest frequency radial mode is likely to be affected by additional sources, such as mixed modes and rotational split components arising from the neighbor $\ell=2$ mode, which are enhanced by the large mode linewidths (see $\mathrm{C} 15$ for more details). To stabilize the fit for this star and provide estimates of the model parameters that are comparable to the other stars in the sample, we have therefore chosen to discard the last measurement of the second difference for this particular target. In the case of KIC 8366239, KIC 9267654, and KIC 10200377, the highest-frequency values are marked as unreliable, according to the Bayesian peak significance test done by $\mathrm{C} 15$, although they were included in the fit since they do not produce any significant change in the results for the same reasons discussed above.

The measurements of the acoustic radius $t_{\mathrm{He}}$ II the amplitude of the signal $A_{\max }$ from Eq. (4), and the corresponding characteristic width $b$, are shown in Fig. 2 (top, middle, and bottom panels, respectively) for all the stars in the sample. We note that while the model parameters (Eq. (3)) and their corresponding $68 \%$ Bayesian credible intervals are estimated by means of DiAmONDS (see Table 1 and Corsaro \& De Ridder 2014 for more details on the derivation of the Bayesian uncertainties), the uncertainties for the additional parameters $t_{\mathrm{He} \mathrm{II}}, A_{\mathrm{max}}$, and $A_{\mathrm{He}}$ were obtained in a subsequent step. In particular, for $A_{\max }$, we used the same sampling of the posterior probability distribution as obtained by DIAMONDS, so we have derived the median and the corresponding $68 \%$ Bayesian credible intervals directly from the marginal probability distributions of $A_{\max }$. For $t_{\mathrm{He} \text { II }}$ and $A_{\mathrm{He}}$, the uncertainties follow from those of the acoustic depth through the definition of the acoustic radius, and by a rescaling of the uncertainties on $A_{\max }$ through Eq. (5), respectively. All the resulting values are listed in Table 1 as well. We note that the precision obtained on our measurements of the acoustic radii of the He II zones is about ten times higher than obtained by Miglio et al. (2010) using CoRoT data. In addition, all the values match those predicted by B14 along the entire range of $v_{\max }$ investigated, showing a clear increasing trend towards lower $v_{\max }$, as expected for more evolved stages of the evolution in the RGB. The derived amplitudes in frequency, $A_{\mathrm{He}}$, are within the range $0.08-0.33 \mu \mathrm{Hz}$, and are varying from star to star with uncertainties on average around $\sim 6 \%$, thus opening the possibility of studying the He abundance by direct comparison with stellar models.

\section{Conclusions}

By exploiting the set of individual mode frequencies extracted by $\mathrm{C} 15$ for a sample of 19 LRGs with a precision level up to $10^{-3} \mu \mathrm{Hz}$, we computed the first differences, Eq. (1), and the 
E. Corsaro et al.: High-precision acoustic helium signatures in 18 low-mass low-luminosity red giants

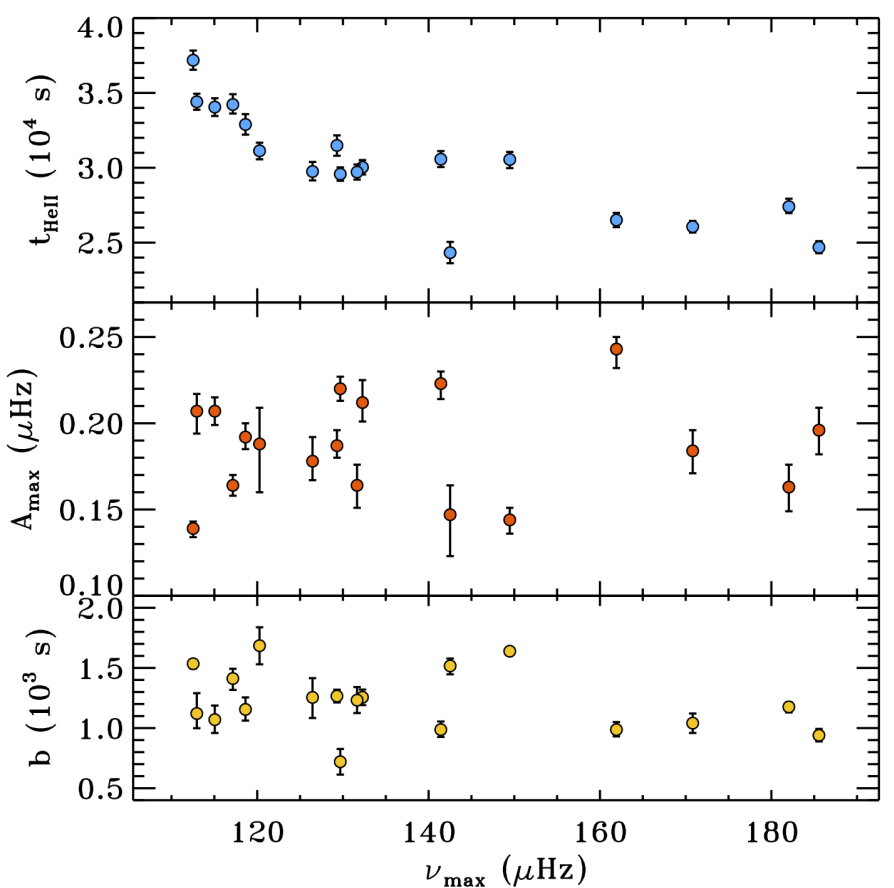

Fig. 2. Acoustic radius of the He II zone (top panel) and corresponding amplitude of the oscillatory signal at $v_{\max }$ (middle panel), and characteristic width $b$ (bottom panel), as a function of $v_{\max }$ for all the stars of the sample. The $68 \%$ Bayesian uncertainties listed in Table 1 are overlaid for all the measurements.

second differences, Eq. (2), for presenting the results on the acoustic glitches of these stars. In this work, we have shown that the acoustic glitches are remarkably clear for all the red giants of the sample, and for both $\ell=0$ and $\ell=2$ modes, up to the second difference (where five to seven different measurements are available for each star, except KIC 10123207, which instead has only four and was not considered in the analysis), with many cases involving $\ell=3$ modes as well.

By adopting the model for the second differences introduced by Houdek \& Gough (2007), Eq. (3), we have extracted the acoustic depth, the characteristic width, and amplitude of the signal of all the He II zones of the stars in our sample (see Table 1) with an unprecedented level of detail for red giant stars (on average $\sim 2 \%$ for the acoustic depths, $\sim 8 \%$ for the characteristic widths, and $\sim 6 \%$ for the amplitudes of the glitch signal in both the second difference and frequency), improved by about one order of magnitude with respect to existing measurements of acoustic depths from the literature. These values, reflecting the high-precision obtained on the individual frequency measurements of the radial modes, confirm the theoretical predictions done by $\mathrm{B} 14$ in the same range of $v_{\max }$. We also stress that the glitch model given by Houdek \& Gough (2007) is able to predict the observations very exhaustively for most of the stars (well within the given uncertainties of the measurements for most of the data points available, as shown in Fig. 1 and in the other figures in the Appendix). This is also supported by our computation of $\sigma_{\text {rms }}$, listed in Table 1, which is at the same precision level as given by the measurements of the second difference for most of the stars analyzed.

Finally, the set of values for $A_{\max }$ and $A_{\mathrm{He}}$ derived in this work, where the latter are not influenced by the position of the glitch, coupled with the high precision achieved, will be useful for investigating the helium content in the envelope of the stars and possibly contributing to study scenarios of helium enrichment in low-mass stars (e.g., see Gratton et al. 2012, and references therein).

Acknowledgements. E.C. is funded by the European Community's Seventh Framework Programme (FP7/2007-2013) under grant agreement $\mathrm{N}^{\circ} 312844$ (SPACEINN). The research leading to these results has received funding from the European Research Council under the European Community's Seventh Framework Programme (FP7/2007-2013) ERC grant agreement $\mathrm{N}^{\circ} 227224$ (PROSPERITY), from the Fund for Scientific Research of Flanders (G.0728.11). E.C. thanks A.-M. Broomhall and A. Miglio for useful discussions.

\section{References}

Baglin, A., Michel, E., Auvergne, M., \& COROT Team. 2006, in Proc. of SOHO 18/GONG 2006/HELAS I, Beyond the spherical Sun, ESA SP, 624, 34 Ballot, J., Turck-Chièze, S., \& García, R. A. 2004, A\&A, 423, 1051 Basu, S. 1997, MNRAS, 288, 572

Basu, S., \& Antia, H. M. 1995, MNRAS, 276, 1402

Beck, P. G., Bedding, T. R., Mosser, B., et al. 2011, Science, 332, 205

Beck, P. G., Montalban, J., Kallinger, T., et al. 2012, Nature, 481, 55

Bedding, T. R., Mosser, B., Huber, D., et al. 2011, Nature, 471, 608

Borucki, W. J., Koch, D., Basri, G., et al. 2010, Science, 327, 977

Broomhall, A.-M., Miglio, A., Montalbán, J., et al. 2014, MNRAS, 440, 1828 (B14)

Christensen-Dalsgaard, J., Monteiro, M. J. P. F. G., Rempel, M., \& Thompson, M. J. 2011, MNRAS, 414, 1158

Christensen-Dalsgaard, J., Silva Aguirre, V., Elsworth, Y., \& Hekker, S. 2014, MNRAS, 445, 3685

Corsaro, E., \& De Ridder, J. 2014, A\&A, 571, A71

Corsaro, E., Fröhlich, H.-E., Bonanno, A., et al. 2013, MNRAS, 430, 2313

Corsaro, E., De Ridder, J., \& García, R. A. 2015, A\&A, in press DOI: $10.1051 / 0004-6361 / 201525895$

Deheuvels, S., García, R. A., Chaplin, W. J., et al. 2012, ApJ, 756, 19

Gough, D. O. 1990, in Lect. Notes Phys., 367, Progress of Seismology of the Sun and Stars, eds. Y. Osaki, \& H. Shibahashi (Berlin: Springer Verlag), 283 Gratton, R. G., Carretta, E., \& Bragaglia, A. 2012, A\&ARv, 20, 50 Houdek, G., \& Gough, D. O. 2007, MNRAS, 375, 861

Koch, D. G., Borucki, W. J., Basri, G., et al. 2010, ApJ, 713, L79

Mazumdar, A., \& Antia, H. M. 2001, A\&A, 368, L8

Mazumdar, A., Michel, E., Antia, H. M., \& Deheuvels, S. 2012, A\&A, 540, A31 Mazumdar, A., Monteiro, M. J. P. F. G., Ballot, J., et al. 2014, ApJ, 782, 18

Miglio, A., Montalbán, J., Carrier, F., et al. 2010, A\&A, 520, L6 Monteiro, M. J. P. F. G., \& Thompson, M. J. 2005, MNRAS, 361, 1187

Monteiro, M. J. P. F. G., Christensen-Dalsgaard, J., \& Thompson, M. J. 2000, MNRAS, 316, 165

Mosser, B., Belkacem, K., Goupil, M.-J., et al. 2010, A\&A, 517, A22

Mosser, B., Barban, C., Montalbán, J., et al. 2011a, A\&A, 532, A86

Mosser, B., Belkacem, K., Goupil, M. J., et al. 2011b, A\&A, 525, L9

Verma, K., Faria, J. P., Antia, H. M., et al. 2014, ApJ, 790, 138

Vorontsov, S. V. 1988, in Advances in Helio- and Asteroseismology, eds. J. Christensen-Dalsgaard \& S. Frandsen, IAU Symp., 123, 151

Vrard, M., Mosser, B., \& Barban, C. 2014, in SF2A-2014: Proc. Annual meeting of the French Society of Astronomy and Astrophysics, eds. J. Ballet, F. Martins, F. Bournaud, R. Monier, \& C. Reylé, 531 


\section{Appendix A: Results for the fitting the acoustic signatures}

All the individual angular frequency measurements and corresponding second differences of the radial modes are listed in the Tables A.1. The results for the first differences $\Delta v_{\ell}(v)$ and second differences $\Delta_{2} v_{\ell}(v)$ of all the LRGs are shown in the Figs. A.1 to A.17, similar to those provided for KIC 12008916 in Fig. 1.

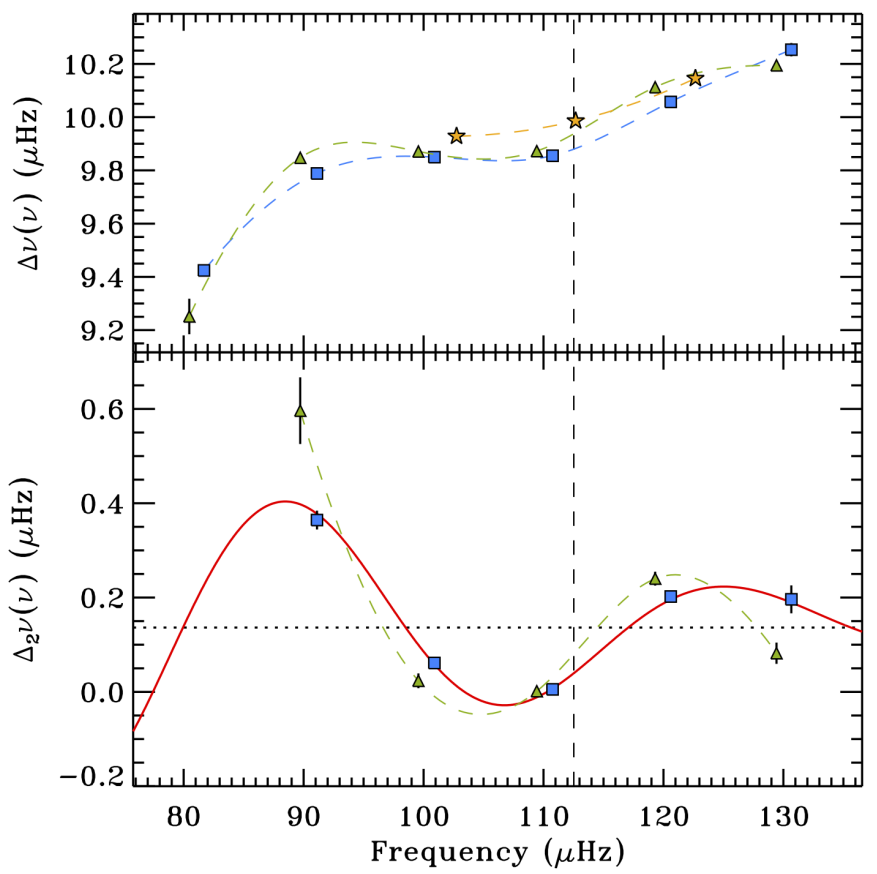

Fig. A.1. Same description as in Fig. 1 but for KIC 3744043, with yellow star from $\ell=3$ modes and corresponding polynomial fit with same color.

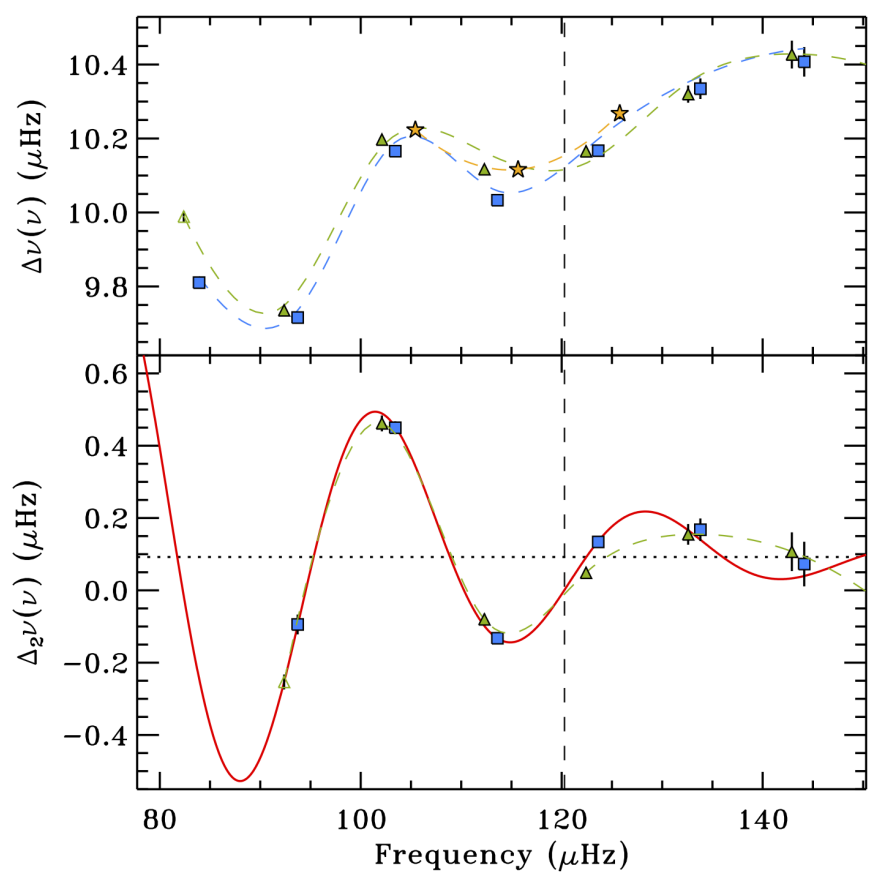

Fig. A.2. Same description as in Fig. 1 but for KIC 6117517, with yellow star from $\ell=3$ modes and corresponding polynomial fit with same color. Open symbols represent measurements that used modes with detection probability under the threshold suggested by $\mathrm{C} 15$.

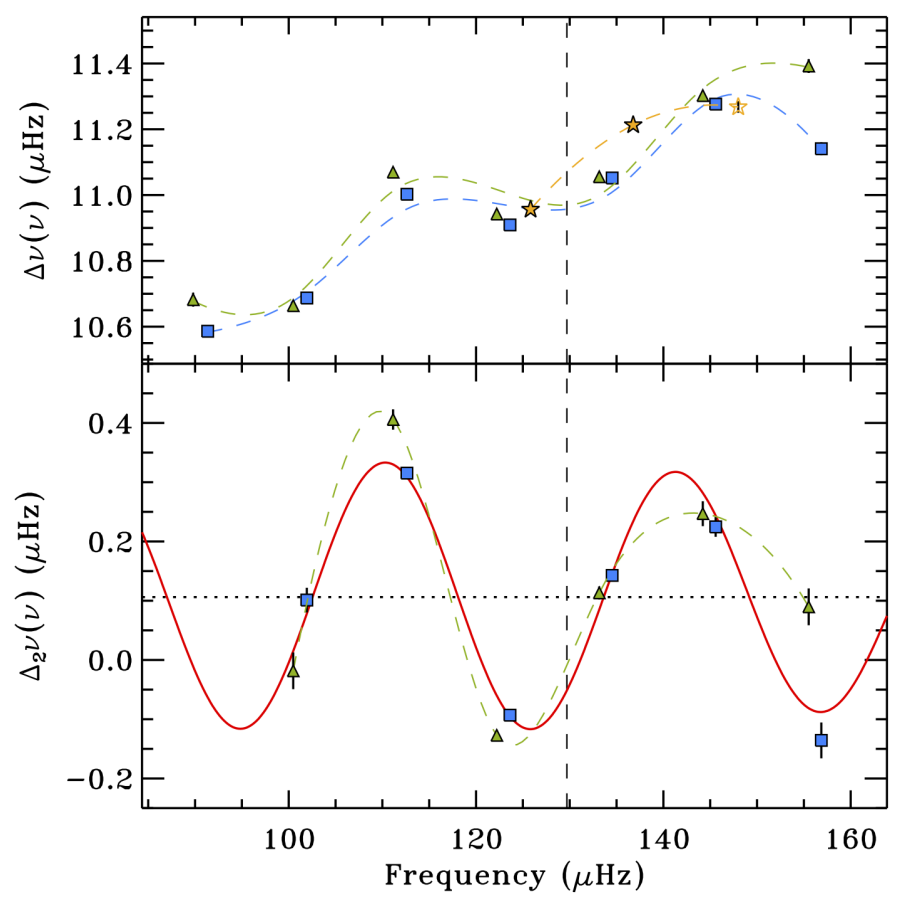

Fig. A.3. Same description as in Fig. 1 but for KIC 6144777, with yellow star from $\ell=3$ modes and corresponding polynomial fit with same color.

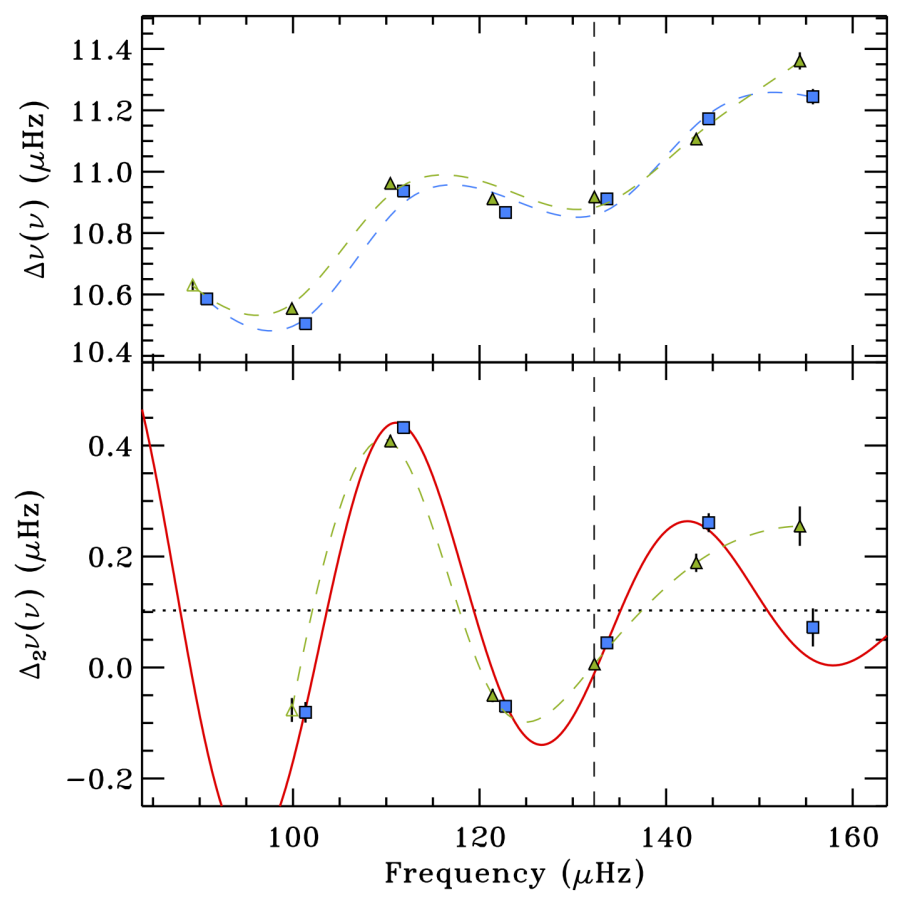

Fig. A.4. Same description as in Fig. 1 but for KIC 7060732. 
E. Corsaro et al.: High-precision acoustic helium signatures in 18 low-mass low-luminosity red giants

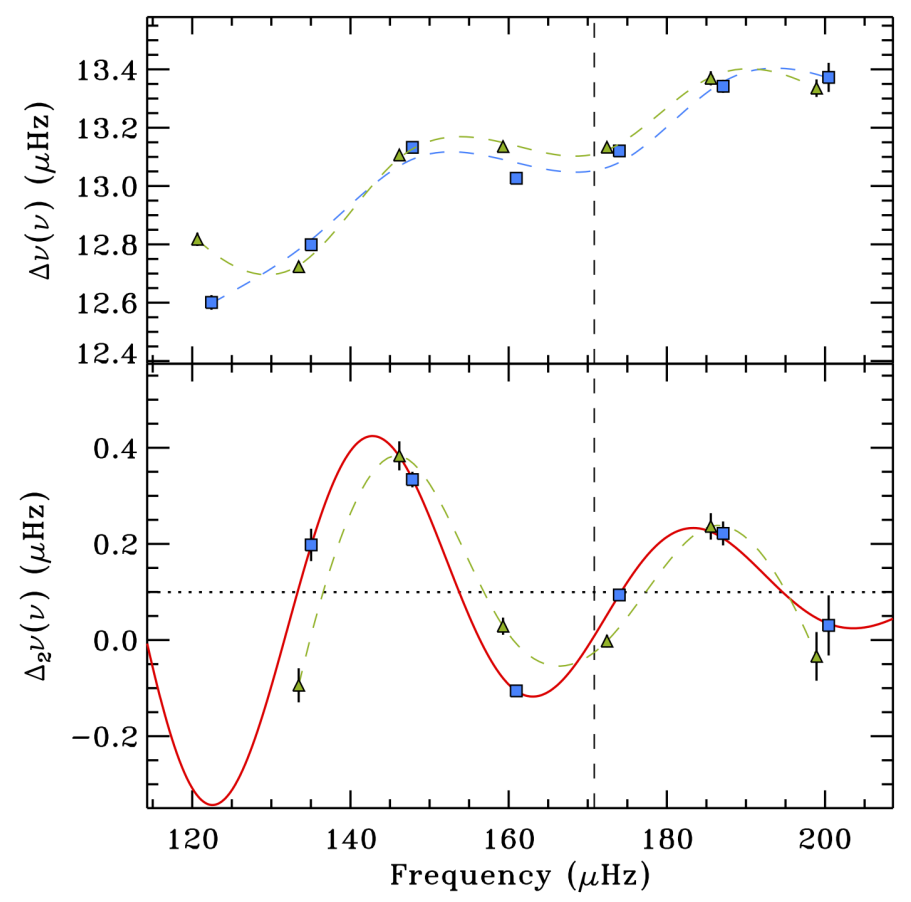

Fig. A.5. Same description as in Fig. 1 but for KIC 7619745.

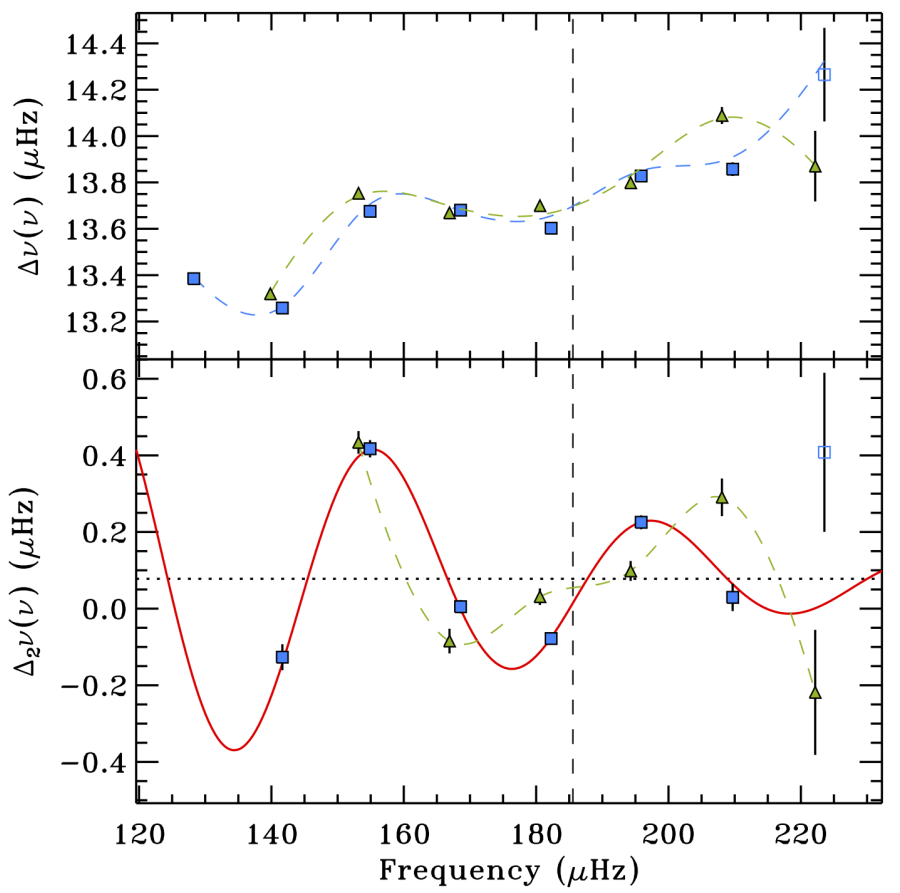

Fig. A.6. Same description as in Fig. 1 but for KIC 8366239. Open symbols represent measurements that used modes with detection probability under the threshold suggested by $\mathrm{C} 15$.

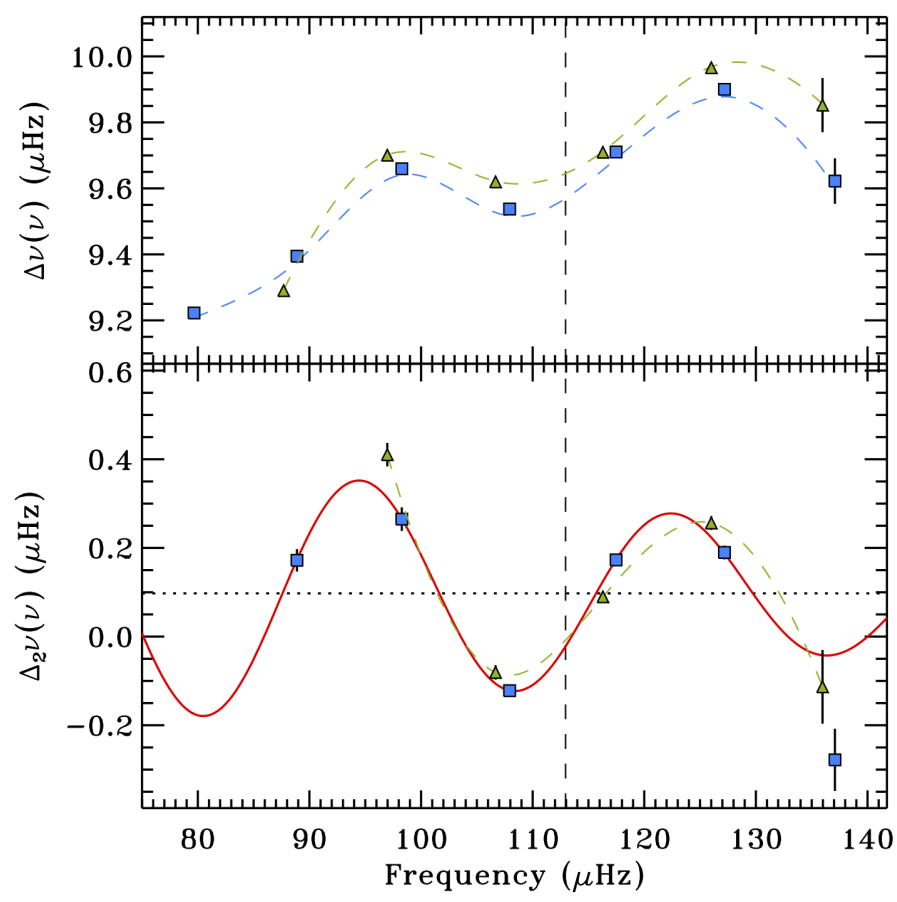

Fig. A.7. Same description as in Fig. 1 but for KIC 8475025.

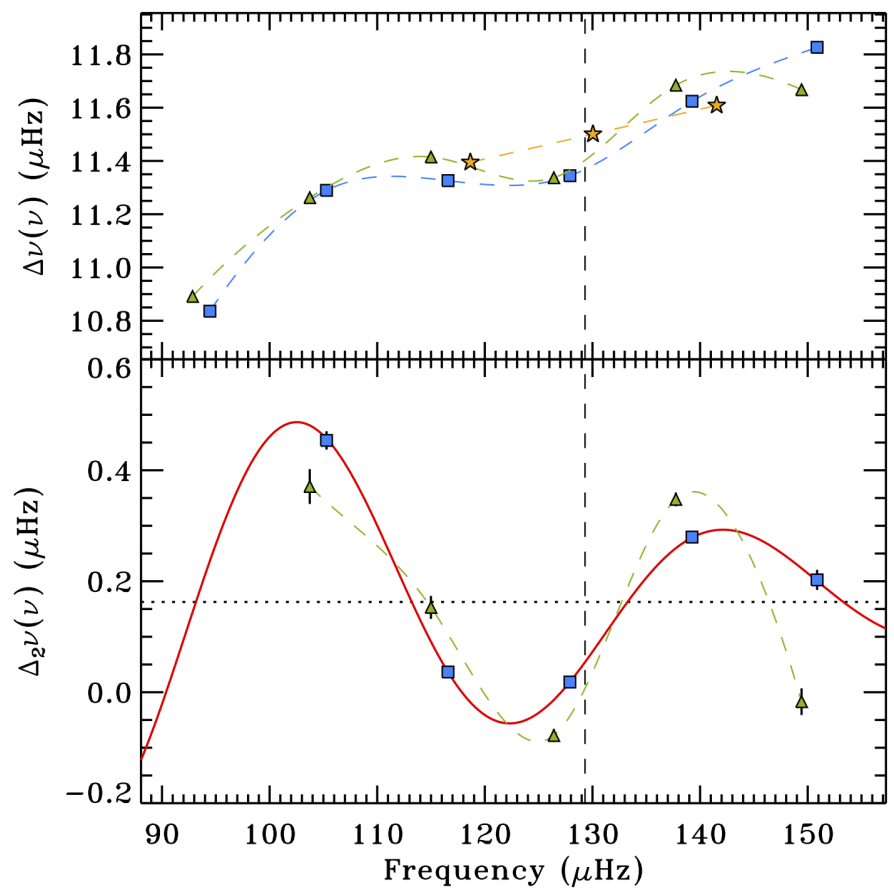

Fig. A.8. Same description as in Fig. 1 but for KIC 8718745, with yellow star from $\ell=3$ modes and corresponding polynomial fit with same color. 


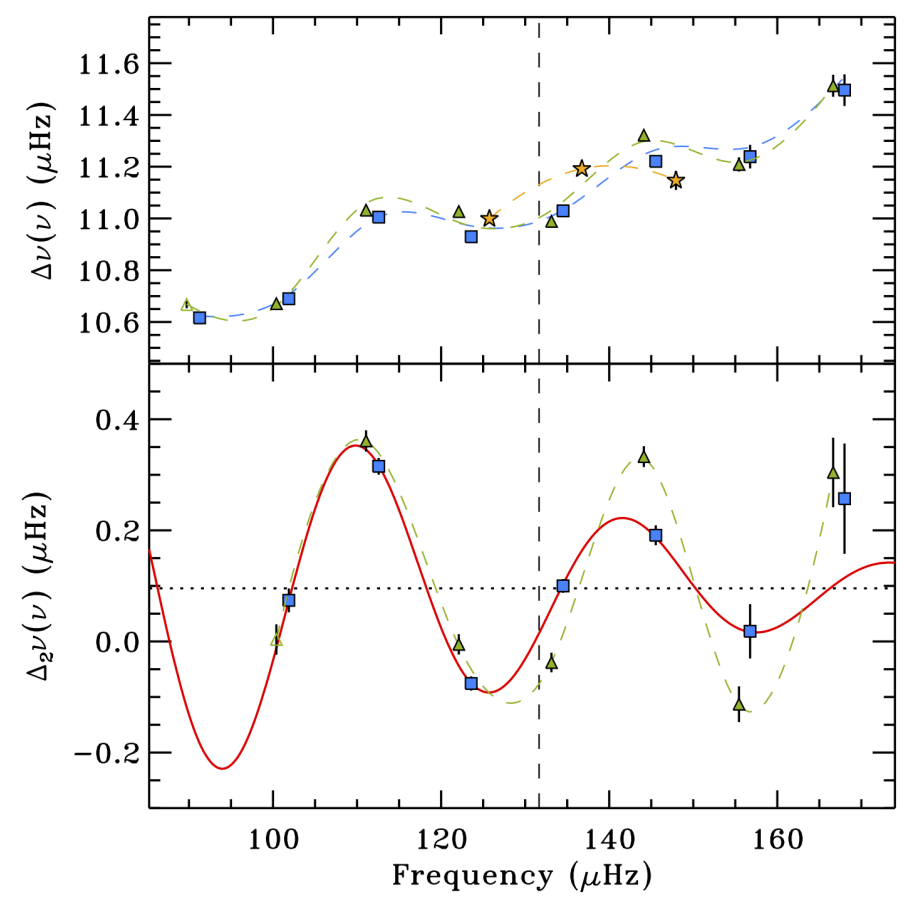

Fig. A.9. Same description as in Fig. 1 but for KIC 9145955, with yellow star from $\ell=3$ modes and corresponding polynomial fit with same color. Open symbols represent measurements that used modes with detection probability under the threshold suggested by $\mathrm{C} 15$.

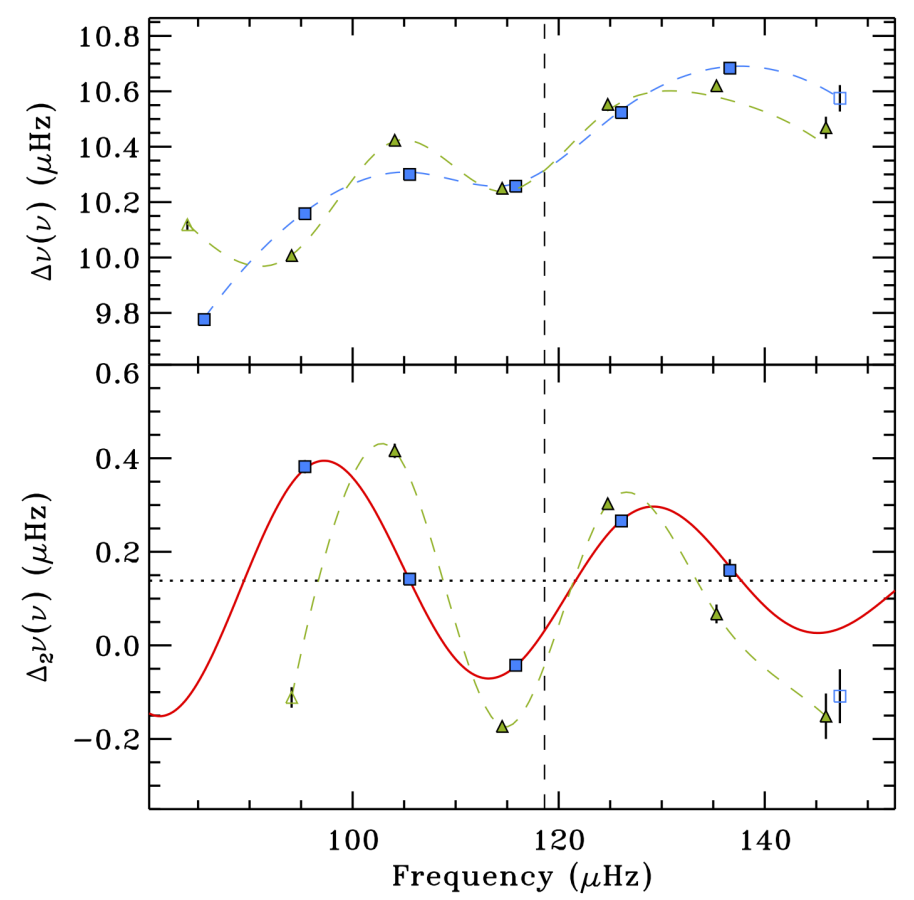

Fig. A.10. Same description as in Fig. 1 but for KIC 9267654. Open symbols represent measurements that used modes with detection probability under the threshold suggested by $\mathrm{C} 15$.

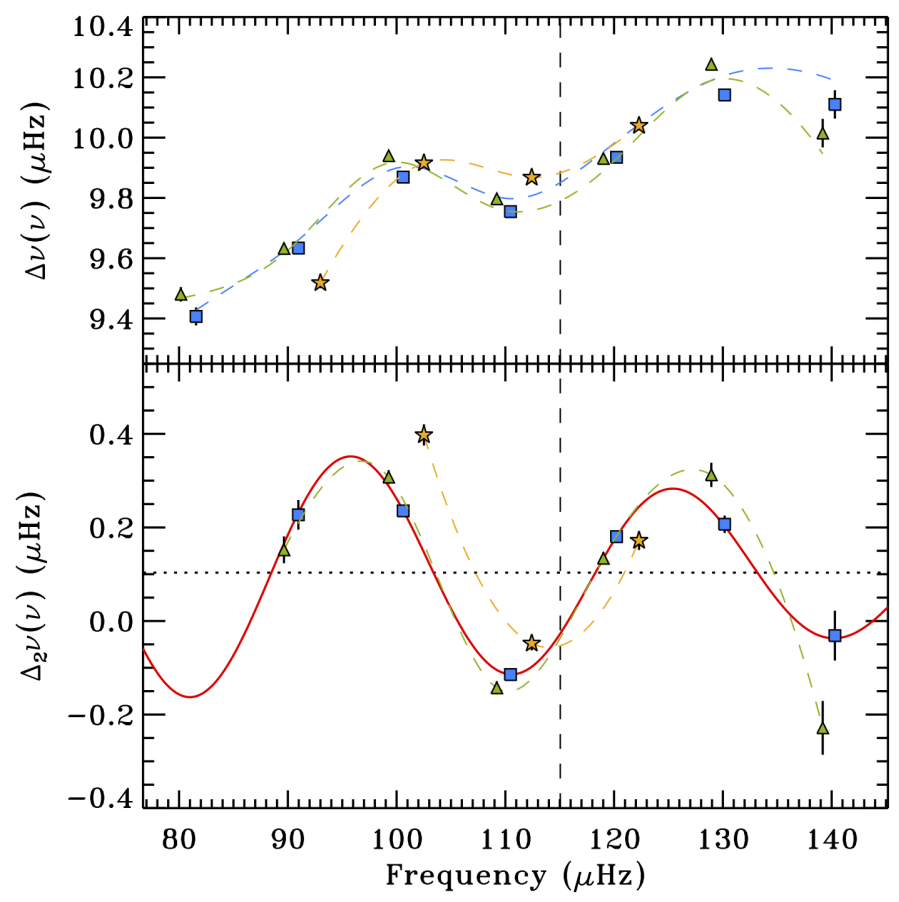

Fig. A.11. Same description as in Fig. 1 but for KIC 9475697, with yellow star from $\ell=3$ modes and corresponding polynomial fit with same color. Open symbols represent measurements that used modes with detection probability under the threshold suggested by $\mathrm{C} 15$.

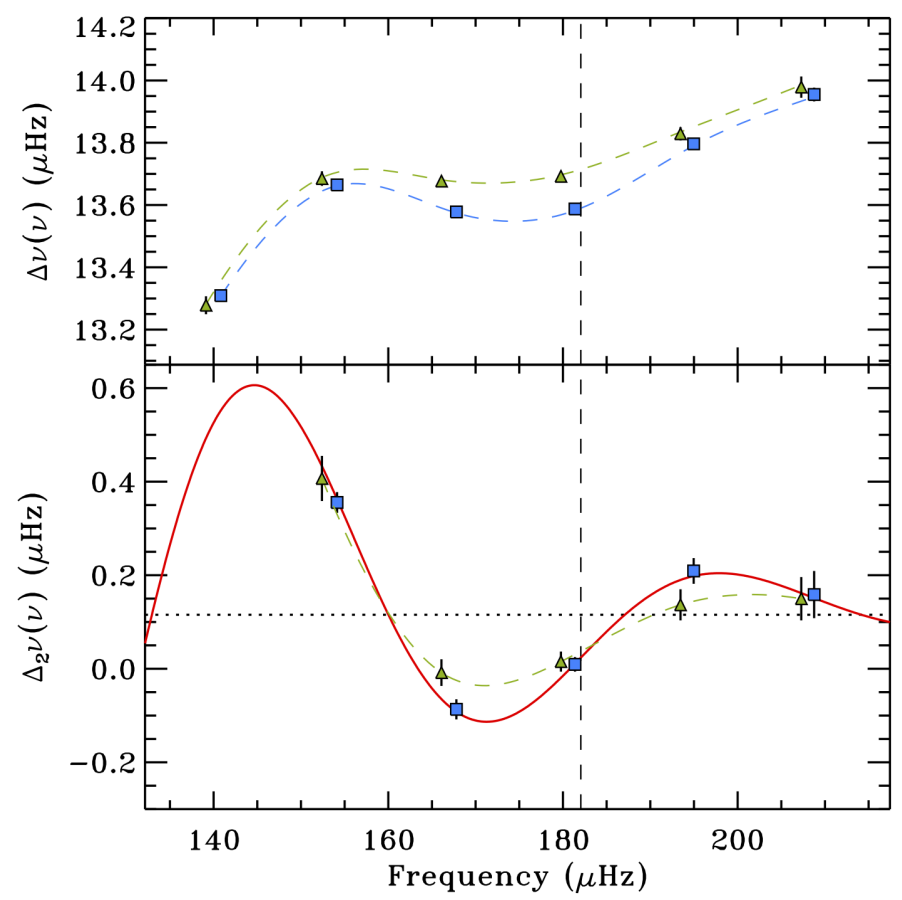

Fig. A.12. Same description as in Fig. 1 but for KIC 9882316. 
E. Corsaro et al.: High-precision acoustic helium signatures in 18 low-mass low-luminosity red giants

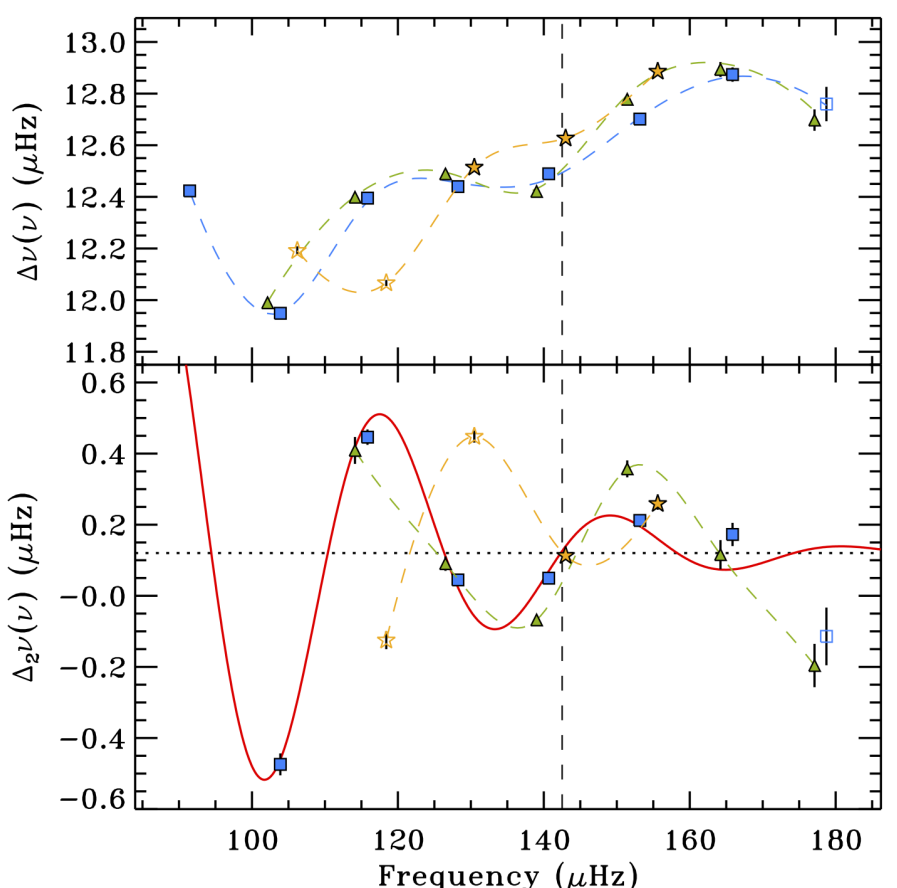

Fig. A.13. Same description as in Fig. 1 but for KIC 10200377, with yellow star from $\ell=3$ modes and corresponding polynomial fit with same color. Open symbols represent measurements that used modes with detection probability under the threshold suggested by $\mathrm{C} 15$.

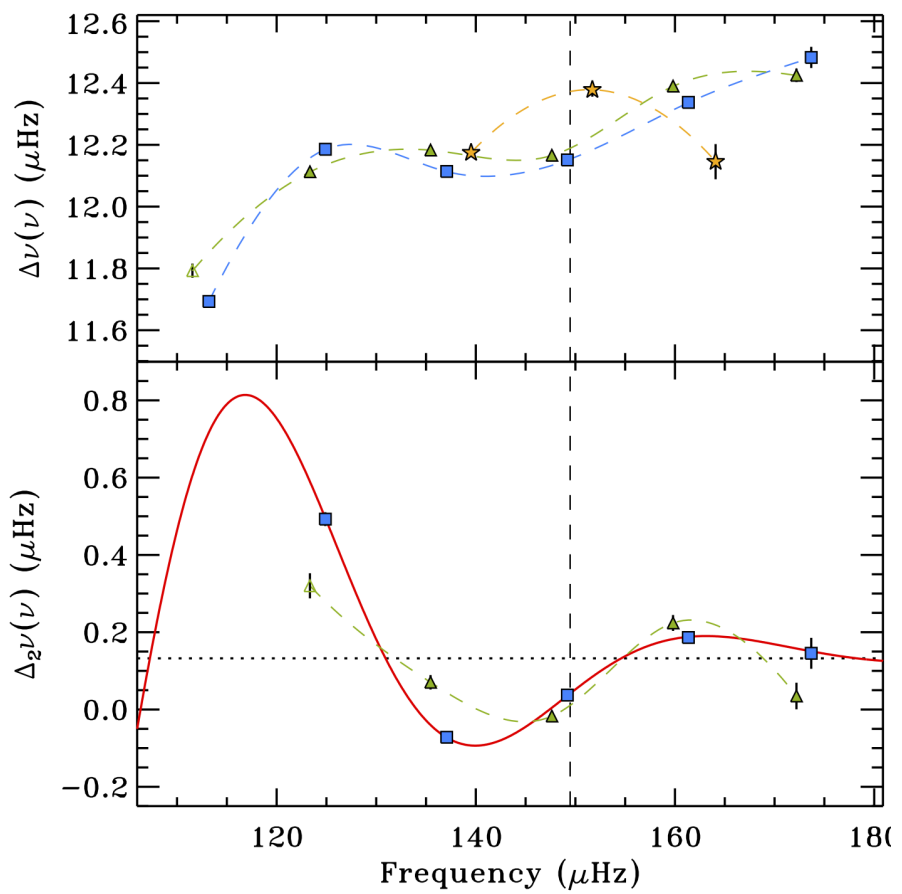

Fig. A.14. Same description as in Fig. 1 but for KIC 10257278, with yellow star from $\ell=3$ modes and corresponding polynomial fit with same color. Open symbols represent measurements that used modes with detection probability under the threshold suggested by $\mathrm{C} 15$.

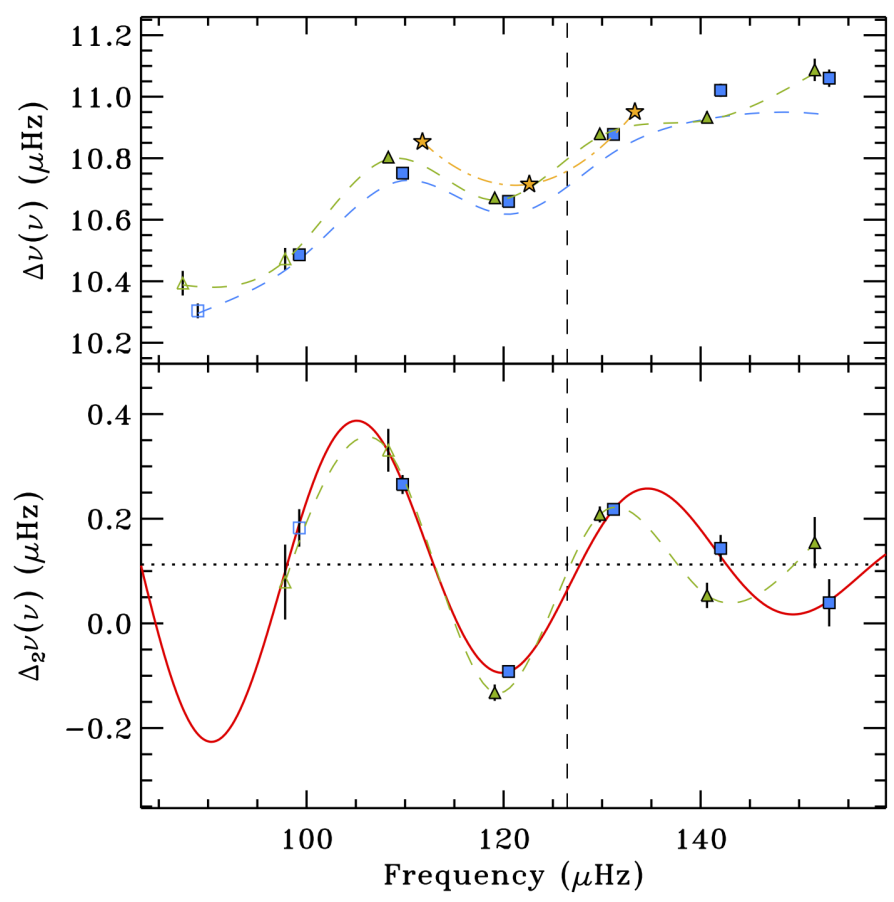

Fig. A.15. Same description as in Fig. 1 but for KIC 11353313, with yellow star from $\ell=3$ modes and corresponding polynomial fit with same color. Open symbols represent measurements that used modes with detection probability under the threshold suggested by $\mathrm{C} 15$.

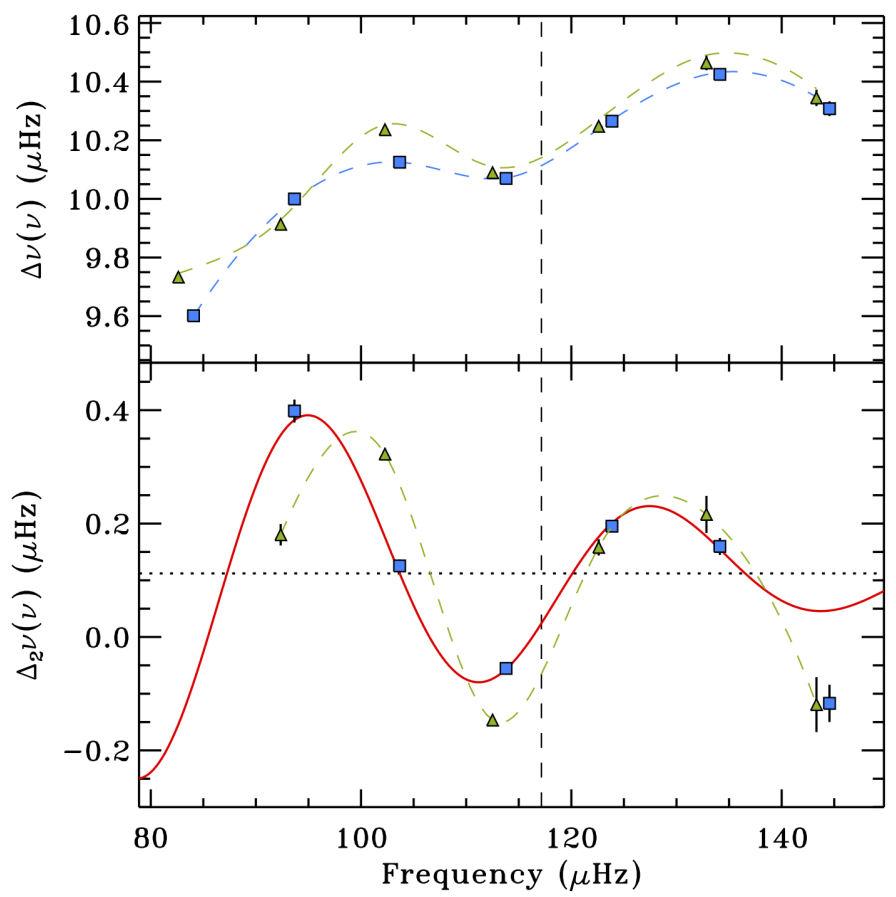

Fig. A.16. Same description as in Fig. 1 but for KIC 11913545. 


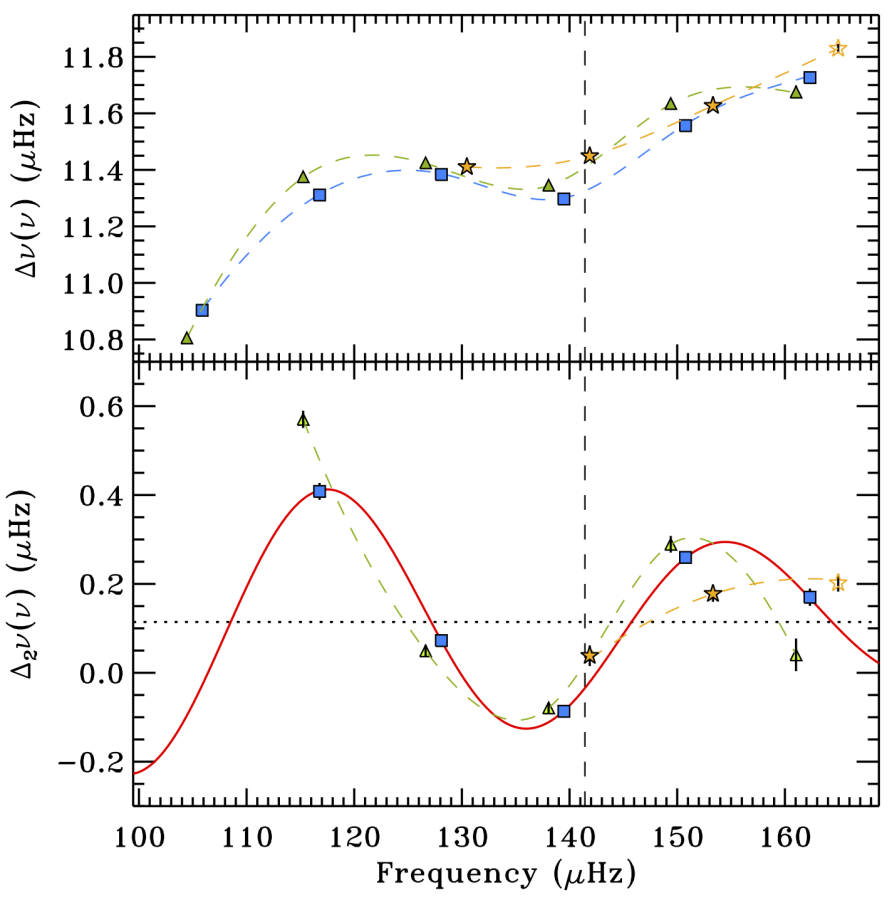

Fig. A.17. Same description as in Fig. 1 but for KIC 11968334, with yellow star from $\ell=3$ modes and corresponding polynomial fit with same color. Open symbols represent measurements that used modes with detection probability under the threshold suggested by $\mathrm{C} 15$.
Table A.1. Radial angular frequencies $\omega_{n, 0}$ and second angular frequency differences $\Delta_{2} \omega_{n, 0}$ for all the stars of the sample.

\begin{tabular}{|c|c|c|}
\hline KIC ID & $\begin{array}{c}\omega_{n, 0} \\
(\mu \mathrm{Hz})\end{array}$ & $\begin{array}{c}\Delta_{2} \omega_{n, 0} \\
(\mu \mathrm{Hz})\end{array}$ \\
\hline 03744043 & $\begin{array}{l}572.48_{-0.05}^{+0.05} \\
633.98_{-0.03}^{+0.03} \\
695.87_{-0.02}^{+0.01} \\
757.79_{-0.02}^{+0.02} \\
820.98_{-0.05}^{+0.06}\end{array}$ & $\begin{array}{l}2.29_{-0.13}^{+0.13} \\
0.39_{-0.08}^{+0.08} \\
0.03_{-0.05}^{+0.05} \\
1.27_{-0.07}^{+0.07} \\
1.23_{-0.19}^{+0.19}\end{array}$ \\
\hline 06117517 & $\begin{array}{l}588.87_{-0.07}^{+0.08} \\
649.91_{-0.04}^{+0.04} \\
713.79_{-0.01}^{+0.01} \\
776.83_{-0.02}^{+0.02} \\
840.71_{-0.05}^{+0.05} \\
905.64_{-0.16}^{+0.17} \\
\end{array}$ & $\begin{array}{r}-0.59_{-0.17}^{+0.17} \\
2.83_{-0.11}^{+0.11} \\
-0.83_{-0.05}^{+0.05} \\
0.84_{-0.06}^{+0.06} \\
1.05_{-0.20}^{+0.20} \\
0.46_{-0.39}^{+0.39} \\
\end{array}$ \\
\hline 06144777 & $\begin{array}{l}640.47_{-0.05}^{+0.05} \\
707.62_{-0.02}^{+0.02} \\
776.75_{-0.01}^{+0.01} \\
845.29_{-0.01}^{+0.01} \\
914.74_{-0.03}^{+0.03} \\
985.59_{-0.08}^{+0.08} \\
\end{array}$ & $\begin{array}{r}0.64_{-0.13}^{+0.13} \\
1.98_{-0.06}^{+0.06} \\
-0.59_{-0.04}^{+0.04} \\
0.90_{-0.04}^{+0.04} \\
1.41_{-0.10}^{+0.10} \\
-0.85_{-0.19}^{+0.19} \\
\end{array}$ \\
\hline 07060732 & $\begin{array}{l}636.76_{-0.05}^{+0.05} \\
702.76_{-0.02}^{+0.02} \\
771.48_{-0.03}^{+0.03} \\
839.76_{-0.02}^{+0.02} \\
908.32_{-0.03}^{+0.03} \\
978.51_{-0.09}^{+0.08} \\
\end{array}$ & $\begin{array}{r}-0.51_{-0.12}^{+0.12} \\
2.72_{-0.07}^{+0.07} \\
-0.44_{-0.07}^{+0.07} \\
0.28_{-0.06}^{+0.06} \\
1.64_{-0.11}^{+0.11} \\
0.45_{-0.22}^{+0.22} \\
\end{array}$ \\
\hline 07619745 & $\begin{array}{l}848.43_{-0.08}^{+0.08} \\
928.84_{-0.03}^{+0.03} \\
1011.36_{-0.02}^{+0.02} \\
1093.21_{-0.02}^{+0.02} \\
1175.64_{-0.04}^{+0.04} \\
1259.48_{-0.14}^{+0.13} \\
\end{array}$ & $\begin{array}{r}1.24_{-0.21}^{+0.21} \\
2.10_{-0.10}^{+0.10} \\
-0.66_{-0.05}^{+0.05} \\
0.59_{-0.06}^{+0.06} \\
1.39_{-0.16}^{+0.16} \\
0.19_{-0.39}^{+0.39} \\
\end{array}$ \\
\hline 08366239 & $\begin{array}{l}890.01_{-0.09}^{+0.09} \\
973.31_{-0.05}^{+0.05} \\
1059.23_{-0.05}^{+0.05} \\
1145.19_{-0.02}^{+0.02} \\
1230.66_{-0.04}^{+0.05} \\
1317.54_{-0.07}^{+0.07} \\
1404.60_{-0.18}^{+0.17} \\
\end{array}$ & $\begin{array}{r}-0.80_{-0.21}^{+0.21} \\
2.62_{-0.14}^{+0.14} \\
0.03_{-0.11}^{+0.11} \\
-0.49_{-0.08}^{+0.08} \\
1.42_{-0.11}^{+0.11} \\
0.18_{-0.22}^{+0.22} \\
2.56_{-1.30}^{+1.30} \\
\end{array}$ \\
\hline 08475025 & $\begin{array}{l}558.46_{-0.06}^{+0.07} \\
617.49_{-0.08}^{+0.08} \\
678.18_{-0.02}^{+0.02} \\
738.10_{-0.02}^{+0.02} \\
799.12_{-0.04}^{+0.04} \\
861.32_{-0.04}^{+0.04} \\
\end{array}$ & $\begin{array}{r}1.08_{-0.16}^{+0.16} \\
1.67_{-0.17}^{+0.17} \\
-0.77_{-0.09}^{+0.09} \\
1.09_{-0.06}^{+0.06} \\
1.19_{-0.10}^{+0.10} \\
-1.75_{-0.44}^{+0.44} \\
\end{array}$ \\
\hline 08718745 & $\begin{array}{l}661.54_{-0.04}^{+0.04} \\
732.48_{-0.03}^{+0.03} \\
803.64_{-0.01}^{+0.01} \\
874.92_{-0.02}^{+0.03} \\
947.96_{-0.04}^{+0.04} \\
\end{array}$ & $\begin{array}{l}2.85_{-0.10}^{+0.10} \\
0.23_{-0.07}^{+0.07} \\
0.12_{-0.05}^{+0.05} \\
1.76_{-0.07}^{+0.07} \\
1.27_{-0.11}^{+0.11} \\
\end{array}$ \\
\hline 09145955 & $\begin{array}{l}640.13_{-0.05}^{+0.06} \\
707.29_{-0.03}^{+0.04} \\
776.44_{-0.04}^{+0.03} \\
845.11_{-0.03}^{+0.03} \\
914.41_{-0.05}^{+0.04} \\
984.91_{-0.06}^{+0.06} \\
1055.53_{-0.28}^{+0.29}\end{array}$ & $\begin{array}{r}0.46_{-0.14}^{+0.14} \\
1.98_{-0.09}^{+0.09} \\
-0.48_{-0.08}^{+0.08} \\
0.63_{-0.08}^{+0.08} \\
1.20_{-0.11}^{+0.11} \\
0.11_{-0.31}^{+0.31} \\
1.61_{-0.62}^{+0.62}\end{array}$ \\
\hline
\end{tabular}

Notes. All the values are reported with $1 \sigma$ uncertainties as obtained by standard error propagation from those provided by $\mathrm{C} 15$. 
E. Corsaro et al.: High-precision acoustic helium signatures in 18 low-mass low-luminosity red giants

Table A.1. continued.

\begin{tabular}{|c|c|c|}
\hline KIC ID & $\begin{array}{c}\omega_{n, 0} \\
(\mu \mathrm{Hz})\end{array}$ & $\begin{array}{l}\Delta_{2} \omega_{n, 0} \\
(\mu \mathrm{Hz})\end{array}$ \\
\hline 09267654 & $\begin{array}{l}599.25_{-0.03}^{+0.03} \\
663.07_{-0.03}^{+0.03} \\
727.79_{-0.01}^{+0.01} \\
792.24_{-0.02}^{+0.02} \\
858.36_{-0.05}^{+0.05} \\
925.49_{-0.12}^{+0.11} \\
\end{array}$ & $\begin{array}{r}2.40_{-0.09}^{+0.09} \\
0.89_{-0.07}^{+0.07} \\
-0.27_{-0.04}^{+0.04} \\
1.67_{-0.06}^{+0.06} \\
1.01_{-0.15}^{+0.15} \\
-0.68_{-0.36}^{+0.36} \\
\end{array}$ \\
\hline 09475697 & $\begin{array}{l}571.57_{-0.04}^{+0.03} \\
632.10_{-0.03}^{+0.03} \\
694.11_{-0.02}^{+0.02} \\
755.40_{-0.01}^{+0.01} \\
817.82_{-0.04}^{+0.04} \\
881.54_{-0.09}^{+0.09} \\
\end{array}$ & $\begin{array}{r}1.43_{-0.20}^{+0.20} \\
1.48_{-0.07}^{+0.07} \\
-0.72_{-0.05}^{+0.05} \\
1.13_{-0.05}^{+0.05} \\
1.30_{-0.12}^{+0.12} \\
-0.20_{-0.33}^{+0.33} \\
\end{array}$ \\
\hline 09882316 & $\begin{array}{l}968.46_{-0.05}^{+0.05} \\
1054.32_{-0.06}^{+0.06} \\
1139.63_{-0.03}^{+0.03} \\
1225.00_{-0.05}^{+0.05} \\
1311.69_{-0.14}^{+0.14} \\
\end{array}$ & $\begin{array}{r}2.23_{-0.14}^{+0.14} \\
-0.54_{-0.13}^{+0.13} \\
0.06_{-0.10}^{+0.10} \\
1.31_{-0.17}^{+0.17} \\
1.00_{-0.32}^{+0.32} \\
\end{array}$ \\
\hline 10200377 & $\begin{array}{l}652.74_{-0.09}^{+0.09} \\
727.81_{-0.05}^{+0.05} \\
805.69_{-0.03}^{+0.03} \\
883.85_{-0.02}^{+0.02} \\
962.33_{-0.02}^{+0.02} \\
1042.13_{-0.06}^{+0.06} \\
1123.02_{-0.17}^{+0.16}\end{array}$ & $\begin{array}{r}-2.98_{-0.19}^{+0.19} \\
2.80_{-0.14}^{+0.14} \\
0.28_{-0.08}^{+0.08} \\
0.31_{-0.05}^{+0.05} \\
1.33_{-0.08}^{+0.08} \\
1.08_{-0.21}^{+0.21} \\
-0.72_{-0.51}^{+0.51}\end{array}$ \\
\hline 10257278 & $\begin{array}{l}784.72_{-0.05}^{+0.05} \\
861.29_{-0.02}^{+0.02} \\
937.40_{-0.02}^{+0.02} \\
1013.75_{-0.02}^{+0.03} \\
1091.27_{-0.07}^{+0.08}\end{array}$ & $\begin{array}{r}3.10_{-0.11}^{+0.11} \\
-0.45_{-0.07}^{+0.07} \\
0.23_{-0.05}^{+0.05} \\
1.17_{-0.09}^{+0.09} \\
0.91_{-0.25}^{+0.25} \\
\end{array}$ \\
\hline 11353313 & $\begin{array}{l}623.65_{-0.09}^{+0.10} \\
689.53_{-0.03}^{+0.03} \\
757.09_{-0.02}^{+0.02} \\
824.06_{-0.02}^{+0.02} \\
892.41_{-0.05}^{+0.05} \\
961.65_{-0.13}^{+0.12}\end{array}$ & $\begin{array}{r}1.15_{-0.22}^{+0.22} \\
1.67_{-0.11}^{+0.11} \\
-0.58_{-0.05}^{+0.05} \\
1.37_{-0.07}^{+0.07} \\
0.90_{-0.16}^{+0.16} \\
0.25_{-0.28}^{+0.28}\end{array}$ \\
\hline 11913545 & $\begin{array}{l}588.52_{-0.06}^{+0.06} \\
651.35_{-0.02}^{+0.02} \\
714.97_{-0.01}^{+0.01} \\
778.24_{-0.01}^{+0.01} \\
842.74_{-0.03}^{+0.03} \\
908.24_{-0.07}^{+0.08}\end{array}$ & $\begin{array}{r}2.50_{-0.13}^{+0.13} \\
0.79_{-0.07}^{+0.07} \\
-0.35_{-0.03}^{+0.03} \\
1.23_{-0.04}^{+0.04} \\
1.00_{-0.09}^{+0.09} \\
-0.74_{-0.21}^{+0.21}\end{array}$ \\
\hline 11968334 & $\begin{array}{l}733.74_{-0.04}^{+0.04} \\
804.81_{-0.03}^{+0.03} \\
876.34_{-0.02}^{+0.01} \\
947.32_{-0.03}^{+0.03} \\
1019.93_{-0.04}^{+0.03}\end{array}$ & $\begin{array}{r}2.56_{-0.12}^{+0.12} \\
0.45_{-0.08}^{+0.08} \\
-0.54_{-0.05}^{+0.05} \\
1.63_{-0.07}^{+0.07} \\
1.07_{-0.12}^{+0.12}\end{array}$ \\
\hline 12008916 & $\begin{array}{l}832.03_{-0.03}^{+0.03} \\
912.25_{-0.04}^{+0.04} \\
993.16_{-0.02}^{+0.02} \\
1073.33_{-0.02}^{+0.03} \\
1155.25_{-0.05}^{+0.05}\end{array}$ & $\begin{array}{r}2.95_{-0.14}^{+0.14} \\
0.69_{-0.09}^{+0.09} \\
-0.74_{-0.06}^{+0.06} \\
1.75_{-0.07}^{+0.07} \\
0.95_{-0.14}^{+0.14}\end{array}$ \\
\hline
\end{tabular}

